\title{
RESULTADOS VISUAIS NO TRATAMENTO CIRÚRGICO DA SUBLUXAÇÃO DE CRISTALINO EM CRIANÇAS
}

\author{
VISUAL OUTCOME AFTER LENSECTOMY FOR SUBLUXATED LENS IN CHILDREN
}

Denise Fornazari de Oliveira ${ }^{1}$;Pollyanna Hueb Marchi² \& Carlos Eduardo Leite Arieta ${ }^{3}$

${ }^{1}$ Pós-graduanda. ${ }^{2}$ Residente. ${ }^{3}$ Docente. Disciplina de Oftalmologia da Faculdade de Ciências Médicas - UNICAMP

CORRESPONDENAIA: Denise Fornazari de Oliveira. Rua Shigeo Mori 303 CEP 13084-080 Campinas, SP. Fone: (19) $3289-9932$

(19) 3236-9002 Fax: (19)3233-6434 e-mail: oliveira@fcm.unicamp.br

OLIVEIRA DF; MARCHI PH \& ARIETA CEL. Resultados visuais no tratamento cirúrgico da subluxação de cristalino em crianças. Medicina, Ribeirão Preto, 35: 62-69, jan./ma r. 2002.

RESUMO: OBJETIVO: descrever os resultados das cirurgias de facectomia via pars plana, realizadas em 40 pacientes (71 olhos) com sudescrevebluxação de cristalino. MATERIAL E MÉTODOS: quarenta pacientes com subluxação de cristalino, submetidos a facectomia-vitrectomia via pars plana, no período de Janeiro de 1990 a Dezembro de 2000, foram avaliados quanto ao diagnóstico sistêmico, doenças oculares associadas, idade do paciente na cirurgia, acuidade visual pré e pós-operatória, e complicações da cirurgia. As indicações para a cirurgia foram acuidade visual menor ou igual a 0,3 ou complicações relacionadas a subluxação.

RESULTADOS: o seguimento pós-operatório variou de 6 a 120 meses (média 42,3 meses). A acuidade visual melhorou em cinqüenta e sete olhos $(80,3 \%)$, e em quarenta e sete olhos $(66,2 \%)$ foi melhor do que 0,5 . Em quatro olhos, a acuidade manteve-se a mesma do préoperatório, e um olho teve piora da acuidade. Complicações ocorreram em dois olhos: um paciente teve descolamento de retina no pós-operatório e outro apresentou sangramento intravítreo no trans-operatório. CONCLUSÕES: Os resultados da série e o tempo de seguimento dos pacientes demonstram que a facectomia-vitrectomia,em um sistema fechado, pode ser um procedimento seguro e efetivo no tratamento da subluxação de cristalino, em crianças, quando o erro refrativo não pode ser corrigido por outros meios, com melhora da acuidade visual e baixo número de complicações.

UNITERMOS: Ectopia do Cristalino. Síndrome de Marfan. Homocistinúria.

\section{INTRODUÇÃO}

A subluxação do cristalino pode ocorrer como manifestação de doença sistêmica, como na síndrome de Marfan, na homocistinúria, na síndrome de WeillMarchesani, na hiperlisinemia, ou em associação com outras doenças oculares, entre elas o glaucoma congênito e a megalocórnea. Também pode ocorrer isoladamente, nos casos de ectopia lentis essencial, ou secundária a trauma ${ }^{(1)}$.

A subluxação progressiva do cristalino induz modificações freqüentes no estado refrativo do olho e a redução da acuidade visual é uma das manifestações oculares mais relevantes ${ }^{(2)}$. A distorção óptica ocorre em casos nos quais o equador da lente se localiza no eixo visual. Além disso, se o cristalino não está completamente deslocado, o relaxamento da zônula pode torná-lo esférico, causando miopia. Há casos em que as distorções da imagem são produzidas pelo astigmatismo decorrente da incidência da luz na periferia e ao lado do cristalino, ou pela falta dele no eixo visual, o que torna o paciente hipermétrope ${ }^{(3,4)}$. Mesmo com cuidadosa refração, nem sempre é possível obter visão satisfatória, com correção para fácico ou afácico. 
A correção cirúrgica, muitas vezes, é a única alternativa viável para a melhora da acuidade visual e prevenção de ambliopia ${ }^{(2)}$. Há algumas décadas, o tratamento cirúrgico das subluxações do cristalino era controverso devido ao alto índice de complicações e resultados visuais insatisfatórios ${ }^{(5)}$. O primeiro cirurgião a descrever uma técnica cirúrgica para cristalinos subluxados foi Dixson, em $1853^{(6)}$. Técnicas cirúrgicas como iridectomia óptica ${ }^{(7)}$, remoção intracapsular ou extracapsular, discisão e aspiração foram des$\operatorname{critas}^{(8)}$. Crioextração e hidroexpressão dos cristalinos subluxados também já foram utilizados ${ }^{(4,9)}$. Estudos demonstravam que perda de vítreo e descolamento de retina ocorriam em muitos pacientes ${ }^{(10)}$.

Peyman $^{(11)}$, em 1979, foi o primeiro a utilizar um instrumento automatizado, em cirurgia intra-ocular com sistema fechado, com abordagem via pars pla$n a$, para cristalinos deslocados. Durante um seguimento de 5 a 45 meses, não foram observadas complicações decorrentes da cirurgia. Outros autores relataram resultados semelhantes ${ }^{(12,13,14 / 18)}$. Com o desenvolvimento da técnica, os resultados apresentaram sensível melhora. Esse sistema mantém as relações anatômicas do olho, não há perda vítrea ou tração da retina, não há dano ao trabeculado e ao endotélio, e a reabilitação visual é rápida ${ }^{(2)}$.

Posteriormente, foi descrita a utilização de anel endocapsular para expansão e estabilização do saco capsular para implante de lente intra-ocular. Entretanto, a utilização do anel, em crianças, ainda é questionável, devido à possibilidade de complicações. O processo inflamatório pós-operatório é mais acentuado nos olhos das crianças do que nos dos adultos e o grau de diálise zonular, em pacientes com subluxação precoce, pode ser maior, além de progressivo ${ }^{(19)}$.

O objetivo deste estudo é descrever os resultados das cirurgias realizadas em 40 pacientes ( 71 olhos) com subluxação de cristalino, no Hospital das Clínicas da Universidade Estadual de Campinas (HC UNICAMP), utilizando-se a técnica de facectomia-vitrectomia via pars plana.

\section{MATERIAL E MÉTODOS}

Foi realizado um estudo retrospectivo, não comparativo, de uma série de casos de crianças e adolescentes portadores de subluxação de cristalino não traumática, atendidos no setor de catarata infantil do ambulatório de Oftalmologia do HC UNICAMP e submetidos a tratamento cirúrgico no período de janeiro de 1990 a dezembro de 2000 . Foram considerados para a avaliação todos os pacientes operados com o diag- nóstico de subluxação do cristalino e que tiveram um seguimento mínimo de seis meses após a realização da cirurgia. Foram incluídos, no estudo, 40 pacientes (71 olhos operados) com idade variando de 1 a 16 anos (média 6,9 anos).

Os pacientes foram submetidos à avaliação oftalmológica antes e depois da cirurgia, que incluiu: acuidade visual com a melhor correção para longe e para perto, quando possível, utilizando-se a Tabela de Snellen, de figuras ou cartões de Teller, biomicroscopia, refração e fundoscopia. A refração foi realizada sob cicloplegia, utilizando-se régua de retinoscopia ou refrator, e, para a fundoscopia, utilizou-se oftalmoscópio indireto. A pressão intra-ocular foi medida com tonômetro de aplanação de Goldman ou Perkins, quando houve colaboração do paciente.

Os critérios adotados para a indicação cirúrgica foram acuidade visual menor ou igual a 20/70 com a melhor correção no olho afetado, opacificação do cristalino, deslocamento do cristalino para a câmara anterior, ou subluxação com a borda do cristalino no eixo visual.

\subsection{Técnica cirúrgica}

Todas as cirurgias e o seguimento pós-operatório foram realizados no Hospital das Clínicas da Universidade Estadual de Campinas. A técnica cirúrgica utilizada foi facectomia e vitrectomia anterior via pars plana. Nos casos de deslocamento do cristalino para a câmara anterior, a cirurgia foi realizada via limbal.

Com o paciente sob anestesia geral e após dilatação da pupila com colírio de tropicamida $1 \%$ e ciclopentolato $1 \%$, realizava-se abertura da conjuntiva e cápsula de Tenon a $3 \mathrm{~mm}$ do limbo temporal superior na subluxação nasal, ou nasal superior na subluxação temporal. Seguia-se esclerotomia paralela e a $3 \mathrm{~mm}$ do limbo, e abertura na câmara anterior para cânula de irrigação, conectada a solução salina balanceada. Feita a abertura da cápsula do cristalino, usando-se lâmina de esclerotomia, em seguida, era realizada a aspiração do córtex e do núcleo, e a remoção do remanescente da cápsula com ponteira com corte de alta velocidade e médio poder de aspiração. Após a vitrectomia anterior, era feita sutura da esclerotomia com um ponto, usando-se Vycril $\square$ 7-0. A incisão corneana, por ser auto-selante, não necessitava de sutura. Ao final, era realizada injeção subconjuntival de dexametasona (2\%) e gentamicina (4\%), na proporção de $1 / 1$.

Em três pacientes, a cirurgia foi realizada via limbal devido à luxação do cristalino para a câmara anterior. A facectomia e vitrectomia foram realizadas segundo a mesma técnica da abordagem via pars pla$n a$, mas com a incisão posicionada no limbo. 
No pós-operatório, utilizou-se colírio de dexametasona $(0,1 \%) 6$ vezes/dia com diminuição gradativa até 30 dias, colírio de antibiótico (tobramicina $0,3 \%$ ) quatro vezes/dia, por 01 semana e colírio midriático (tropicamida 1\%) uma vez por dia, durante 15 dias. Foram realizadas avaliações no $1^{\circ}, 7^{\circ}, 15^{\circ}, 30^{\circ}$ e $60^{\circ}$ dias de pós-operatório, e, após, periodicamente de acordo com a evolução.

A correção óptica (óculos ou lentes de contato) foi prescrita entre sete e 15 dias após a cirurgia. Terapia oclusiva foi realizada de acordo com a acuidade visual e a fixação binocular.

Os dados obtidos foram tabulados e avaliados quanto a: diagnóstico sistêmico, doenças oculares associadas, idade do paciente na cirurgia, acuidade visual pré e pós-operatória, refração pré e pós-operatória e complicações trans e pós-operatórias.

\section{RESULTADOS}

A Tabela I resume os dados referentes ao diagnóstico e idade dos pacientes no momento da indicação da cirurgia. Foi feito diagnóstico de síndrome de Marfan em 18 pacientes, ectopia lentis simples em 17 pacientes, e seis apresentaram outros diagnósticos: homocistinúria em três casos, microesferofacia em um caso, síndrome de Stickler em um caso e síndrome de Weill Marchesani em um caso.

Em quatro pacientes (oito olhos - 11,26\%), foi diagnosticada opacidade do cristalino subluxado, e três pacientes $(7,5 \%)$ apresentavam megalocórnea.

Dos 40 pacientes incluídos no estudo, vinte (50\%) foram submetidos à cirurgia após os cinco anos de idade. Somente três pacientes $(7,5 \%)$ foram operados antes dos três anos de vida.

Foram submetidos à cirurgia em ambos os olhos 31 pacientes, e nove em apenas um olho. O tempo de seguimento pós-operatório variou de 6 a 120 meses (média 42,3 meses).

Dos 71 olhos operados, 47 $(66,2 \%)$ obtiveram acuidade visual de 0,5 ou mais, oito olhos $(11,3 \%)$ tiveram acuidade visual final maior ou igual a 0,3 e menor que 0,5 , e 57 olhos $(80,3 \%)$ tiveram melhora da acuidade visual (Tabela II). Em quatro olhos (5,6\%), de quatro pacientes, a acuidade visual permaneceu a mesma do pré-operatório. Em dois pacientes, isso resultou de ambliopia, um apresentou glaucoma congênito as- sociado a subluxação e um paciente tinha outras alterações oculares, decorrentes da subluxação: sinéquias anteriores e edema de córnea na área de toque endotelial, que não regrediu totalmente após a cirurgia. Um paciente apresentou descolamento de retina em um olho $(1,3 \%)$ no terceiro mês de pós-operatório, e, após a retinopexia, permaneceu com percepção luminosa. Os resultados estão apresentados nas Tabelas III (Síndrome de Marfan), IV (ectopia lentis simples) e V (outros diagnósticos).

Em nove olhos (12,6\%), de cinco pacientes, não foi possível medir a acuidade visual antes da cirurgia, por falta de colaboração do paciente. Dentre eles, cinco olhos tiveram acuidade visual final maior ou igual a 0,3 , e quatro olhos tiveram acuidade visual final maior ou igual a 0,5 .

Nos pacientes com síndrome de Marfan, a acuidade visual melhorou em 28 olhos (90,3\%), permaneceu a mesma em dois olhos, por provável ambliopia, $\mathrm{e}$ piorou em um paciente (um olho) que apresentou descolamento de retina no pós-operatório. Em 20 olhos $(64,5 \%)$, a acuidade foi maior ou igual a 0,5 (Tabela III).

Nos pacientes com ectopia simples, 20 olhos $(68,9 \%)$ tiveram melhora da acuidade visual. Nessa série, em sete pacientes, não foi possível obter a acuidade visual pré-operatória, mas, três olhos tiveram acuidade visual final maior ou igual a 0,3 . Os pacientes com outros diagnósticos apresentaram acuidade visual melhor ou igual a 0,5 em 9 olhos $(81,8 \%)$.

Em 16 olhos (22,5\%), a acuidade visual final ficou abaixo de 0,3 , mas, sete olhos tiveram melhora de visão, e quatro olhos mantiveram a visão medida no pré-operatório.

\begin{tabular}{|c|c|c|c|c|}
\hline \multicolumn{5}{|c|}{$\begin{array}{l}\text { Tabela I: Descrição dos casos segundo diagnóstico, número de } \\
\text { olhos e idade na ocasião da cirurgia de facectomia via pars plana. } \\
\text { Hospital das Clínicas, Universidade Estadual de Campinas - } 1990 \\
\text { a } 2000 \text {. }\end{array}$} \\
\hline $\begin{array}{c}\text { Características da } \\
\text { amostra }\end{array}$ & Marfan & $\begin{array}{l}\text { Ectopia } \\
\text { simples }\end{array}$ & Outros ${ }^{*}$ & Total \\
\hline Número & 18 & 16 & 6 & 40 \\
\hline Número de olhos & 31 & 29 & 11 & 71 \\
\hline \multicolumn{5}{|c|}{ Idade na ciruraia (anos): } \\
\hline$<3$ & 1 & 2 & - & 3 \\
\hline $3-5$ & 5 & 9 & 3 & 17 \\
\hline$>5$ & 12 & 5 & 3 & 20 \\
\hline
\end{tabular}




\begin{tabular}{|c|c|c|c|c|c|c|c|c|}
\hline \multirow[b]{2}{*}{$\begin{array}{c}\text { Acuidade Visual } \\
\text { (AV) }\end{array}$} & \multicolumn{2}{|c|}{ Marfan } & \multicolumn{2}{|c|}{ Ectopia simples } & \multicolumn{2}{|c|}{ Outros ${ }^{*}$} & \multicolumn{2}{|c|}{ Total } \\
\hline & $\begin{array}{l}\text { Antes } \\
f(\%)\end{array}$ & $\begin{array}{c}\text { Depois } \\
f(\%)\end{array}$ & $\begin{array}{l}\text { Antes } \\
f(\%)\end{array}$ & $\begin{array}{c}\text { Depois } \\
f(\%)\end{array}$ & $\begin{array}{l}\text { Antes } \\
f(\%)\end{array}$ & $\begin{array}{c}\text { Depois } \\
f(\%)\end{array}$ & $\begin{array}{l}\text { Antes } \\
f(\%)\end{array}$ & $\begin{array}{c}\text { Depois } \\
f(\%)\end{array}$ \\
\hline $0,5 \geq A V$ & $1(3,2)$ & $20(64,5)$ & - & $18(62,1)$ & - & $9(81,8)$ & $1(1,4)$ & $47(66,2)$ \\
\hline $0,3 \leq \mathrm{AV}<0,5$ & $3(9,7)$ & $5(16,1)$ & $1(3,4)$ & $2(6,9)$ & $1(9,1)$ & $1(9,1)$ & $5(7,1)$ & $8(11,3)$ \\
\hline$A V<0,3$ & $27(87,1)$ & $6(19,4)$ & $21(72,4)$ & $9(31,0)$ & $8(72,7)$ & $1(9,1)$ & $56(78,8)$ & $16(22,5)$ \\
\hline Sem medida AV & - & - & $7(24,2)$ & - & $2(18,2)$ & - & $9(12,7)$ & - \\
\hline TOTAL & \multicolumn{2}{|c|}{31} & \multicolumn{2}{|c|}{29} & \multicolumn{2}{|c|}{11} & \multicolumn{2}{|c|}{71} \\
\hline $\begin{array}{l}\mathrm{N}^{\circ} \text { de olhos com } \\
\text { melhora da } \mathrm{AV}\end{array}$ & \multicolumn{2}{|c|}{$28(90,3)$} & \multicolumn{2}{|c|}{$20(68,9)$} & \multicolumn{2}{|c|}{$9(81,8 \%)$} & \multicolumn{2}{|c|}{$57(80,3 \%)$} \\
\hline \multicolumn{9}{|c|}{$\begin{array}{l}\text { *adidade visual corriqida com óculos ou lentes de contato. Medida realizada com tabela de Snellen, tabelas de fiquras ou } \\
\text { cartốes de Teller, de acordo com a idade do paciente. } \\
\text { * S. Weill Marchesani, S. Stickler, homocistinúria, microesterofacia. }\end{array}$} \\
\hline
\end{tabular}

Dois pacientes (dois olhos $-2,6 \%$ ) tiveram complicações relacionadas à cirurgia: um apresentou sangramento intravítreo durante a cirurgia, que regrediu espontaneamente, e o outro apresentou descolamento de retina três meses após a cirurgia.

Em 34 pacientes, foram utilizados óculos para correção da afacia e, em seis pacientes, lentes de contato.

\section{COMENTÁRIOS}

A ectopia de cristalino vem sendo tratada com cirurgia há longo tempo e discute-se o momento correto da indicação e da técnica a ser utilizada. Em crianças, o principal fator a ser considerado é a ambliopia. Se esta não pode ser tratada com óculos ou lentes de contacto, a cirurgia é a melhor opção ${ }^{(1)}$. Observou-se, na série de casos citados neste trabalho, que $50 \%$ dos pacientes foram submetidos à cirurgia após os cinco anos de idade. A indicação da cirurgia é mais comum quando a borda do cristalino atinge o eixo visual, o que ocorre progressivamente. No entanto, não há justificativa para aguardar até que a criança tenha mais idade para a realização da cirurgia e a cirurgia precoce pode prevenir a ambliopia ${ }^{(12)}$.

A possibilidade de usar vitrectomia com sistema fechado permite maior segurança nas indicações precoces de cirurgia, nos casos de subluxações. A utilização dessa técnica permite melhor controle transoperatório, diminuindo consideravelmente os riscos de complicações e possibilitando melhor reabilitação visual $^{(12,13,20,21)}$.
A técnica cirúrgica de escolha para os pacientes estudados foi a facectomia via pars plana, exceto em três casos, quando foi utilizada a via limbal, nos casos de luxação para a câmara anterior. Apesar de não haver estudos comparando as duas técnicas cirúrgicas em olhos de crianças, não foram observadas diferenças nos resultados apresentados por vários autores, utilizando-se uma ou outra técnica ${ }^{(1,22)}$.

Os resultados visuais, na série de casos aqui relatados, foram consistentes com outros já apresentados (Tabela VI): a acuidade visual melhorou em $80,3 \%$ dos casos e $66,2 \%$ tiveram acuidade final melhor ou igual a 0,5 . Os resultados visuais apenas foram limitados por ambliopia, uma vez que ocorreram complicações somente em dois olhos (2,6\%). Halpert, em $1996^{(2)}$, encontrou $88 \%$ de melhora na acuidade visual; Salehpour e colaboradores ${ }^{(1)}$ descreveram melhora em $92 \%$ dos casos operados.

Os pacientes com Síndrome de Marfan, 25 olhos $(80,64 \%)$, apresentaram, no pós-operatório, hipermetropia de 10 dioptrias ou maior, os pacientes com ectopia lentis simples, 20 olhos (69\%), e os pacientes com outros diagnósticos quatro olhos $(36,36 \%)$, o que é compatível com um comprimento axial próximo do normal. Em alguns pacientes, houve diminuição dos valores da hipermetropia pré-operatória, e outros apresentaram mudanças na refração, passando da alta miopia para valores acima de 10 dioptrias positivas. Isso ocorreu, provavelmente, porque os erros refracionais do pré-operatório eram decorrentes das alterações induzidas pelo deslocamento da lente e não por alterações no comprimento axial. 


\begin{tabular}{|c|c|c|c|c|c|c|}
\hline \multicolumn{7}{|c|}{$\begin{array}{l}\text { Tabela III: Descrição dos casos com diagnó stico de Síndrome de Marfan. Hospital das Clínicas, Universidade } \\
\text { de Campinas - } 1990 \text { a } 2000 .\end{array}$} \\
\hline $\begin{array}{l}\text { Doença Ocular } \\
\text { associada }\end{array}$ & $\begin{array}{l}\text { Idade } \\
\text { Ciruraia } \\
\text { (anos) }\end{array}$ & Av Préop.(1) & $\begin{array}{l}\text { Refração Pré } \\
\text { Op.(1) }\end{array}$ & $\begin{array}{l}\text { Av Pós- } \\
\text { Op.(2) }\end{array}$ & $\begin{array}{l}\text { Refração Pós } \\
\text { Op.(2) }\end{array}$ & $\begin{array}{l}\text { Tempo } \\
\text { Seq. (3) } \\
\text { (meses) }\end{array}$ \\
\hline 1 & $\begin{array}{l}3 \\
7\end{array}$ & $\begin{array}{l}0,5 \\
0,1\end{array}$ & 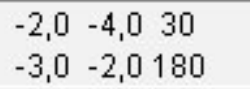 & $\begin{array}{l}0,9 \\
1,0\end{array}$ & $\begin{array}{l}+12,75-0,7520 \\
+13,25\end{array}$ & 55 \\
\hline 2 & $\begin{array}{l}8 \\
8\end{array}$ & $\begin{array}{l}0,1 \\
0,4\end{array}$ & $\begin{array}{l}+6,0 \\
-12,0\end{array}$ & 0,8 & $\begin{array}{l}+11,0-1,0180 \\
+11,0\end{array}$ & 48 \\
\hline microcórnea. & $\begin{array}{l}10 \\
10\end{array}$ & $\begin{array}{l}\mathrm{CD}(4) \\
\mathrm{CD}\end{array}$ & $\begin{array}{l}+12,0 \\
+12,0\end{array}$ & $\begin{array}{l}0,3 \\
0,3\end{array}$ & $\begin{array}{l}+12,50 \\
+12,50\end{array}$ & 55 \\
\hline 4 & $\begin{array}{l}8 \\
8\end{array}$ & $\begin{array}{l}\mathrm{CD} \\
\mathrm{CD}\end{array}$ & $\begin{array}{l}-8,0 \\
-8,0\end{array}$ & $\begin{array}{l}1,0 \\
0,9\end{array}$ & $\begin{array}{l}+11,50-1,7515 \\
+11,75\end{array}$ & 60 \\
\hline 5 & 3 & $\begin{aligned} & 0,3 \\
< & 0,1\end{aligned}$ & $\begin{array}{l}+15,5-1,075 \\
+16,75-0,5\end{array}$ & $\begin{array}{l}0,9 \\
0,1\end{array}$ & $\begin{array}{l}+13,50 \\
+14,0\end{array}$ & 72 \\
\hline 6 & 6 & $\begin{array}{l}0,1 \\
0,4\end{array}$ & $\begin{array}{lll}-6,0 & & \\
-2,0 & -2,5 & 180\end{array}$ & $\begin{array}{l}0,1 \\
\text { nẫo op(5) }\end{array}$ & $+11,0-0,510$ & 7 \\
\hline megaloc órnea & $\begin{array}{l}4 \\
4\end{array}$ & $\begin{array}{l}\mathrm{CD} \\
\mathrm{CD}\end{array}$ & $\begin{array}{l}+14,5-2,0180 \\
+14,50\end{array}$ & $\begin{array}{l}1,0 \\
0,1\end{array}$ & $\begin{array}{l}+12,25 \\
+11,75\end{array}$ & 67 \\
\hline 8 & $\begin{array}{l}16 \\
16\end{array}$ & $\begin{array}{l}\mathrm{CD} \\
\mathrm{CD}\end{array}$ & $\begin{array}{l}+13,50 \\
+14,50\end{array}$ & $\begin{array}{l}0,4 \\
\mathrm{CD}\end{array}$ & $\begin{array}{l}+15,5-1,0 \quad 110 \\
+15,0\end{array}$ & 24 \\
\hline 9 & $\begin{array}{l}11 \\
11\end{array}$ & $\begin{array}{l}0,2 \\
0,3\end{array}$ & $\operatorname{IMP}(6)$ & $\begin{array}{l}0,5 \\
0,8\end{array}$ & $\begin{array}{l}+15,0 \\
+15,0\end{array}$ & 60 \\
\hline 10 & $\begin{array}{l}4 \\
4\end{array}$ & $\begin{array}{l}C D \\
0,2\end{array}$ & $\begin{array}{l}+19,0 \\
+10,0\end{array}$ & $\begin{array}{l}0,4 \\
0,8\end{array}$ & $\begin{array}{l}+16,0 \\
+16,0 \quad-1,5 \quad 80\end{array}$ & 21 \\
\hline 11 & $\begin{array}{l}10 \\
10\end{array}$ & $\begin{array}{l}0,1 \\
0,1\end{array}$ & $\begin{array}{llll}-4,0 & -2,0 & 180 \\
-5,0 & -2,0 & 180\end{array}$ & $\begin{array}{l}\text { nẫo op } \\
0,8\end{array}$ & 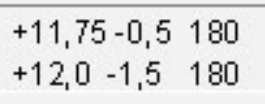 & 31 \\
\hline catarata & $\begin{array}{l}4 \\
4\end{array}$ & $\begin{array}{l}0,2 \\
0,2(t)\end{array}$ & IMP & $\begin{array}{l}1,0 \\
1,0\end{array}$ & $\begin{array}{l}+10,75-1.2515 \\
+11,25-1,5 \quad 160\end{array}$ & 53 \\
\hline 13 & $\begin{array}{c}10 \\
9\end{array}$ & $\begin{array}{l}0,2 \\
0,1\end{array}$ & $\begin{array}{l}+13,0 \\
+13,0\end{array}$ & $\begin{array}{l}0,5 \\
0,9\end{array}$ & 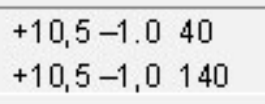 & $\begin{array}{l}24 \\
39\end{array}$ \\
\hline 14 & 6 & $\begin{array}{l}\mathrm{CD} \\
0,5\end{array}$ & $\begin{array}{l}+15,0 \\
+15,0\end{array}$ & $\begin{array}{l}0,2 \\
\text { nẩo op }\end{array}$ & $\begin{array}{l}+15,5-0,5120 \\
+15,25\end{array}$ & 50 \\
\hline 15 & $\begin{array}{l}7 \\
8\end{array}$ & $\begin{array}{l}0,1 \\
0,2\end{array}$ & $\begin{array}{l}-19,0 \\
-19,0\end{array}$ & $\begin{array}{l}1,0 \\
1,0\end{array}$ & $\begin{array}{l}+6,0 \\
+4,5\end{array}$ & $\begin{array}{l}20 \\
10\end{array}$ \\
\hline catarata & $\begin{array}{l}11 \\
11\end{array}$ & $\begin{array}{l}0,2 \\
0,2\end{array}$ & $\begin{array}{l}-13,0 \\
-10,5\end{array}$ & $\begin{array}{l}0,8 \\
1,0\end{array}$ & $\begin{array}{l}+6,0-0,75180 \\
+8,0-1,0 \quad 180\end{array}$ & 18 \\
\hline 17 & 15 & $\begin{array}{l}0,1 \\
0,1\end{array}$ & $\begin{array}{l}-7,25 \\
-7,00\end{array}$ & $\begin{array}{l}0,4 \\
\text { nẫo op }\end{array}$ & $+13,25-1,0100$ & 6 \\
\hline 18 & $\begin{array}{l}3 \\
3\end{array}$ & $\begin{aligned} & 0,2 \\
\Leftrightarrow & 0,1(t)(7)\end{aligned}$ & $\begin{array}{l}-6,0 \\
-6,0\end{array}$ & $\begin{array}{l}\text { nã̃o op } \\
\mathrm{PL}(8)\end{array}$ & & 24 \\
\hline
\end{tabular}




\begin{tabular}{|c|c|c|c|c|c|c|c|}
\hline Caso & Doenca ocular & $\begin{array}{l}\text { Idade ciruraia } \\
\text { (anos) }\end{array}$ & $\begin{array}{l}\text { Av pré } \\
\text {-op.(1) }\end{array}$ & $\begin{array}{c}\text { Refracẫo pré- } \\
\text { op.(1) }\end{array}$ & $\begin{array}{l}\text { Av pós- } \\
\text { op.(2) }\end{array}$ & $\begin{array}{c}\text { Refracẫo pós } \\
\text { op.(2) }\end{array}$ & $\begin{array}{c}\text { Tempo } \\
\text { seq.(3) } \\
\text { (meses) }\end{array}$ \\
\hline 1 & meqalocórnea & $\begin{array}{l}3 \\
3\end{array}$ & $N(4)$ & $\operatorname{lMP} P(5)$ & $\begin{array}{l}0.2 \\
0.8\end{array}$ & $\begin{array}{r}+8.5-0.55 \\
+9.0-0.5155\end{array}$ & 120 \\
\hline 2 & & 5 & NI & IMP & $\begin{array}{c}0.2 \\
\text { nẫo op(6) }\end{array}$ & $+8,0-2,0120$ & 75 \\
\hline 3 & meqalocórnea & $\begin{array}{l}13 \\
13\end{array}$ & $\begin{array}{l}0.2 \\
0.1\end{array}$ & $\begin{array}{r}+4,0 \\
+3,0\end{array}$ & $\begin{array}{c}\text { nẫo op } \\
0.1\end{array}$ & $\begin{array}{l}+4.50 \\
+5.0\end{array}$ & 120 \\
\hline 4 & & $\begin{array}{l}2 \\
6\end{array}$ & $\begin{array}{c}\mathrm{CD} \\
\mathrm{CD}(7)\end{array}$ & $\begin{array}{c}+7.0-3.010 \\
+7.0\end{array}$ & $\begin{array}{l}0.3 \\
0.2\end{array}$ & $\begin{array}{l}+7.0-3.0 \quad 10 \\
+7.0-1.0 \quad 180\end{array}$ & 29 \\
\hline 5 & & $\begin{array}{l}7 \\
7\end{array}$ & $\begin{array}{l}\mathrm{CD} \\
0.2\end{array}$ & $\begin{array}{l}-10,0 \\
-4,0\end{array}$ & $\begin{array}{l}0.9 \\
0.9\end{array}$ & $\begin{array}{r}+16.0-1.5180 \\
+16.0-1.55\end{array}$ & 72 \\
\hline 6 & $\begin{array}{l}\text { sinéq anterior } \\
\text { edema córnea }\end{array}$ & $\begin{array}{l}3 \\
3\end{array}$ & NI & IMP & $\begin{array}{l}0.2 \\
0.2\end{array}$ & $\begin{array}{l}+14.0 \\
+16.0\end{array}$ & 28 \\
\hline 7 & & $\begin{array}{l}5 \\
5\end{array}$ & NI & IMP & $\begin{array}{l}0.4 \\
0.8\end{array}$ & 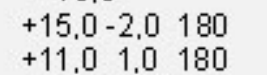 & 29 \\
\hline 8 & & 2 & $\begin{array}{c}\leq 0.1 \\
0.8 \text { (t) }(8)\end{array}$ & $\begin{array}{l}\operatorname{IMP} P \\
+2,0\end{array}$ & $\begin{array}{c}0.1 \\
\text { nẫo op }\end{array}$ & $+13.0-3.5180$ & 19 \\
\hline 9 & catarata & $\begin{array}{l}7 \\
7\end{array}$ & $\begin{array}{l}0,1 \\
0,2\end{array}$ & $\begin{array}{l}+18,50 \\
+16,0\end{array}$ & $\begin{array}{l}0.8 \\
0.8\end{array}$ & $\begin{array}{l}+13,0-1,0180 \\
+12,0\end{array}$ & 48 \\
\hline 10 & & $\begin{array}{l}4 \\
4\end{array}$ & $\begin{array}{l}0.2 \\
0.2\end{array}$ & $\begin{array}{l}+13,0 \\
+13,0\end{array}$ & $\begin{array}{l}1.0 \\
1.0\end{array}$ & $\begin{array}{r}+15.0-1.7525 \\
+15.0-1.25150\end{array}$ & 96 \\
\hline 11 & & $\begin{array}{l}5 \\
5\end{array}$ & $\begin{array}{c}<0.1 \\
0.1\end{array}$ & $\begin{array}{r}+13,0 \\
+12,25\end{array}$ & $\begin{array}{l}0.8 \\
0.5\end{array}$ & $\begin{array}{l}+13.75-1.520 \\
+13.00-1.0150\end{array}$ & 24 \\
\hline 12 & & $\begin{array}{l}16 \\
16\end{array}$ & $\begin{array}{l}\mathrm{CD} \\
0.1\end{array}$ & $\begin{array}{c}+9.5-1.55 \\
+11.0\end{array}$ & $\begin{array}{l}0.1 \\
0.9\end{array}$ & $\begin{array}{r}+9.50-1.75110 \\
+10.25-2.0175\end{array}$ & 34 \\
\hline 13 & & $\begin{array}{l}5 \\
5\end{array}$ & $\begin{array}{l}0.3 \\
0.2\end{array}$ & $\begin{array}{l}-5,0 \\
-8,0\end{array}$ & $\begin{array}{l}0.8 \\
0.8\end{array}$ & $\begin{array}{l}+8.5 \\
+9.75-1.0150\end{array}$ & 24 \\
\hline 14 & & $\begin{array}{l}3 \\
3\end{array}$ & $\begin{array}{c}0.2 \\
0.1(t)\end{array}$ & $\operatorname{IMP}$ & $\begin{array}{c}0.8 \\
0.8(t)\end{array}$ & $\begin{array}{ll}+12,0-0,5 & 180 \\
+12,0-0,5 & 180\end{array}$ & 7 \\
\hline 15 & & 5 & 0.2 & $-21,0$ & 0.2 & $+14.5-1.75180$ & \\
\hline & & 5 & 0.2 & $-20,0$ & 0.5 & $+14,25-1,010$ & 12 \\
\hline 16 & & $\begin{array}{l}11 \\
11\end{array}$ & $\begin{array}{l}0,1 \\
0,1\end{array}$ & $\begin{array}{l}-10,0-2,590 \\
-10,0-2,5 \quad 90\end{array}$ & $\begin{array}{l}0.8 \\
0.5\end{array}$ & $\begin{array}{l}+12.0-1.75175 \\
+13.25-1.520\end{array}$ & 12 \\
\hline
\end{tabular}

\begin{tabular}{|c|c|c|c|c|c|c|c|c|}
\hline Caso & $\begin{array}{l}\text { Diaq. (1) } \\
\text { sistêmico }\end{array}$ & Doenca ocular & $\begin{array}{l}\text { Idade } \\
\text { cirurcia } \\
\text { (anos) }\end{array}$ & $\begin{array}{c}\text { Av } \\
\text { pré-op. } \\
(2)\end{array}$ & $\begin{array}{l}\text { Refracẫo } \\
\text { pré op. (2) }\end{array}$ & $\begin{array}{c}\text { Av } \\
\text { pósop. } \\
\text { (3) }\end{array}$ & $\begin{array}{l}\text { Refracẫo } \\
\text { pós op.(3) }\end{array}$ & $\begin{array}{l}\text { Tempo seq. } \\
\text { (4) (meses) }\end{array}$ \\
\hline 1 & Homoc & & 13 & $\begin{array}{l}0.1 \\
0.4\end{array}$ & $\begin{array}{l}-8,0 \\
-8,0-3,0 \quad 50\end{array}$ & $\begin{array}{c}0.2 \\
\text { nẫo op(5) }\end{array}$ & $+13,0-1,0180$ & 18 \\
\hline 2 & Homoc & $\begin{array}{l}\text { luxacão câmara } \\
\text { anterior }\end{array}$ & $\begin{array}{l}4 \\
4\end{array}$ & $N(6)$ & $\operatorname{lm} P(7)$ & $\begin{array}{l}0.9 \\
0.5\end{array}$ & $\begin{array}{l}+11.0-1.0180 \\
+12.25-1.520\end{array}$ & 72 \\
\hline 3 & Homoc & microesferofacia & $\begin{array}{l}8 \\
8\end{array}$ & $\begin{array}{l}0,3 \\
0,2\end{array}$ & $\begin{array}{l}-10,0-5.0180 \\
-9.75-5,040\end{array}$ & $\begin{array}{l}0.9 \\
0.8\end{array}$ & $\begin{array}{l}+11,0-3.5180 \\
+9.5-2,0180\end{array}$ & 66 \\
\hline 4 & S. Stickler & catarata & $\begin{array}{l}5 \\
5\end{array}$ & $\begin{array}{c}0,2 \\
\mathrm{CD}(8)\end{array}$ & $\begin{array}{l}-14,0-3,070 \\
-15,0-1,5105\end{array}$ & $\begin{array}{l}1.0 \\
0.8\end{array}$ & $\begin{array}{l}+4.5 \\
+3.75\end{array}$ & 36 \\
\hline 5 & $\begin{array}{l}\text { S.Weill } \\
\text { Marchesani }\end{array}$ & catarata & $\begin{array}{l}11 \\
11\end{array}$ & $\begin{array}{l}<0.1 \\
<0.1\end{array}$ & $\begin{array}{l}-29.5 \\
-28,0 \\
\end{array}$ & $\begin{array}{l}0.5 \\
0.4\end{array}$ & $\begin{array}{l}+7.0-1.545 \\
+8.0-1.0150\end{array}$ & 43 \\
\hline 6 & & microesferofacia & $\begin{array}{l}3 \\
3\end{array}$ & $\begin{array}{c}0.1 \\
0.2(t)(9)\end{array}$ & $\begin{array}{l}-6,0 \\
-5,0 \\
\end{array}$ & $\begin{array}{l}0.5 \\
0.8\end{array}$ & $\begin{array}{l}+6.0-2.0180 \\
+8,0-1.5180\end{array}$ & 36 \\
\hline
\end{tabular}




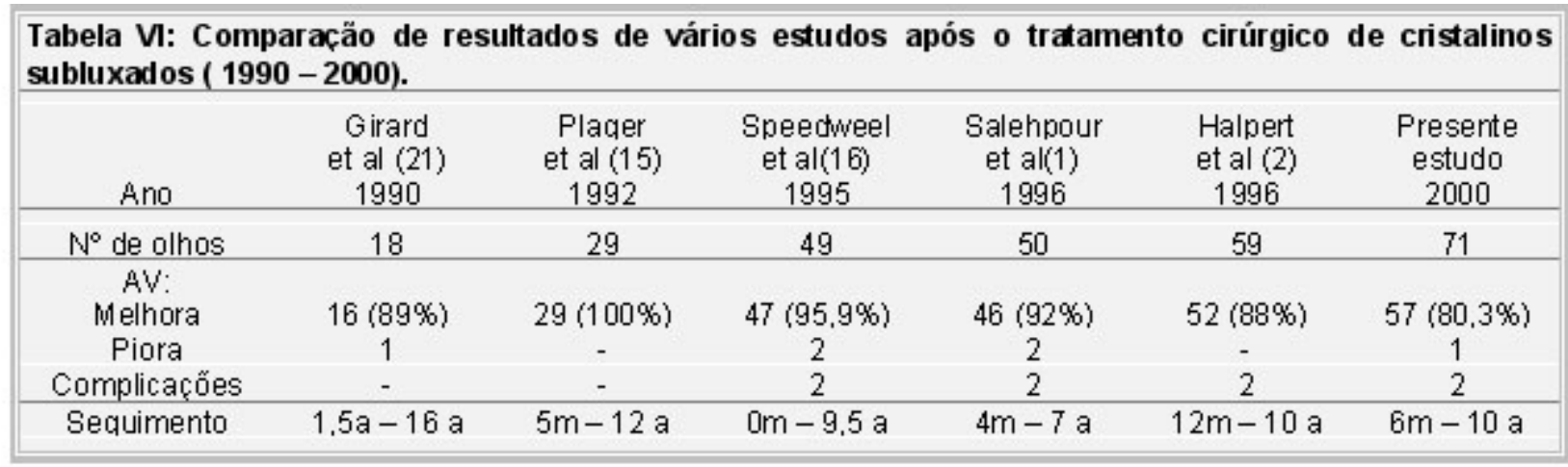

Nessa série, ocorreram complicações em dois olhos de dois pacientes. Um paciente, com diagnóstico de síndrome de Marfan, apresentou descolamento de retina no terceiro mês de pós-operatório, que é uma das mais temidas complicações. Entretanto, deve ser ressaltado que o descolamento da retina, nos pacientes com síndrome de Marfan, pode ocorrer mesmo com condutas conservadoras ${ }^{(2)}$.

A correção pós-operatória com óculos tem-se mostrado o método mais adequado para a correção da afacia desses pacientes. Apesar das distorções ópticas periféricas, causadas pelos óculos, estes são de adaptação mais fácil, tanto para as crianças quanto para os pais, de menor custo e mais seguros. As lentes de contato exigem maior colaboração das crianças, envolvimento dos pais, além de trocas freqüentes.

A utilização de implantes intra-oculares para a correção da afacia, na cirurgia da subluxação, tem sido descrita com fixação escleral ${ }^{(23,24)}$, lentes de câmara anterior ${ }^{(25)}$, ou, ainda, com anel de tensão endocapsular ${ }^{(19,26)}$. Entretanto, implantes desse tipo, em crianças, ainda são pouco utilizados devido ao potencial de complicações que podem representar no pós-operatório, pelas características próprias do olho da criança: proliferação epitelial secundária e contração da cápsula são mais pronunciadas, e o grau de diálise zonular, em pacientes com subluxação precoce, pode ser mais acentuado que em adultos. Como o tamanho do anel endocapsular é desenhado para adultos, rasgos radiais podem ocorrer pois o saco capsular de crianças é pequeno para o implante ${ }^{(19)}$. Modificações no anel poderão melhorar o uso em cirurgias infantis.

Os resultados obtidos no presente estudo, concordantes com outros já apresentados, confirmam a efetividade da técnica cirúrgica em relação aos resultados visuais e à baixa ocorrência de complicações. A facectomia via pars plana permanece como uma alternativa relativamente segura a ser considerada no tratamento da subluxação de cristalino, em crianças, quando não for mais possível a correção do erro refracional por outros meios.

OLIVEIRA DF; MARCHI PH \& ARIETA CEL. Vlisual outcome after lensectomy for subluxated lens in children. Medicina, Ribeirão Preto, 35: 62-69, jan./mar. 2002.

ABSTRACT: PURPOSE: To describe the results of surgical treatment for subluxated lens in forty patients (seventy-one eyes) using lensectomy. METHODS: Forty patients with subluxated lens that were submitted to pars plana lensectomy from January 1990 to December 2000 were evaluated regarding systemic diagnosis, related ocular disease, age of patient at surgery, pre and post operative visual acuity and complications related to surgery. Indications for surgery were best corrected visual acuity of 20/70 or less and complications related to dislocated lens. RESULTS: Best corrected visual acuity improved in fifty seven eyes $(80,3 \%)$, there was no change in visual acuity in four eyes $(5,6 \%)$ and visual acuity became worse than before in one eye $(2.08 \%)$. Follow up ranged from 6 to 120 months. Complications occurred in two eyes: one had post-operative retinal detachment and another had intraoperative vitreous hemorrhage. CONCLUSIONS: The results and long-term followup for these patients demonstrate that lensectomy as a closed-system for subluxated lens improves visual acuity and seems to be a safe procedure with few complications.

UNITERMS: Ectopia Lentis. Marfan Syndrome, Homocystinuria 


\section{REFERÊNCIAS BIBLIOGRÁFICAS}

1 - SALEHPOUR O; LAVY T; LEONARD J \& TAYLOR D. The surgical management of non traumatic ectopic lens. J Pediatr Ophthalmol Strabismus 33:8-13, 1996.

2 - HALPERT M \& BEN EZRA D. Surgery of hereditary subluxated lens in children. Ophthalmology 103:681-686, 1996.

3 - NELSON LB \& MAUMENEE I. Ectopia lentis. Surv Ophthalmol 27:143-160, 1982.

4 - BLUMENTHAL M; KURTZ S \& ASSIA EI. Hydroexpression of subluxated lenses using a glide. Ophthalmic Surg 25: 3437, 1994.

5 - JARRET WH. Dislocations of the lens: a study of 166 hospitalized cases. Arch Ophthalmol 78:289-296, 1967.

6 - DIXON J. On the treatment of posterior displacement of the lens. Lancet 2:313, 1853.

7 - VARGA B. The results of my operations improving visual acuity of ectopia lentis. Ophthalmologica 162: 98-110, 1971.

8 - MAUMENEE AE \& RYAN SJ. Aspiration technique in the management of the dislocated lens. Am J Ophthalmol 68:808-811, 1969.

9 - CROLL M \& CROLL LS. Cryoextraction of dislocated lenses. Ann Ophthalmol 7:1245-1252, 1975.

10 - ZEEMAN WPC. Ectopia lentis congenital. Acta Ophthalmol 20:1-13, 1942

11 - PEYMAN GA; RAUCHAND M; GOLDBERG MF \& RITACIAD. Management of subluxated and dislocated lenses with the vitriophage. Br J Ophthalmol 63:771-778, 1979.

12 - BEHKI R; NOEL LP \& CLARKE WN. Limbal lensectomy in the management of ectopia lentis in children. Arch Ophthalmol 108:809-811, 1990.

13 - SEETNER AA \& CRAWFORD JS. Surgical correction of lens dislocation in children. Am J Ophthalmol 91:106-110,1981.

14 - REESE PD \& WEIGEIST TA. Pars plana management of ectopia lentis in children. Arch Ophthalmol 105:1202-1204, 1987.

15 - PLAGER DA; PARKS MM; HELVESTON EM \& ELLIS FD. Surgical treatment for subluxated lenses in children. Ophthalmology 99:1018-1023, 1992.
16 - SPEEDWELL L \& RUSSEL-EGGITT I. Improvement in visual acuity in children with ectopia lentis. J Pediatr Ophthalmol Strabismus 32:94-97, 1995.

17 - FRIEDMAN Z \& FEINER M. A simplified technique for extraction of subluxated lenses in young patient. Ophthalmic Surg Lasers 29:949-950, 1998.

18 - HAKIN KN; JACOBS M; ROSEN P; TAYLOR D \& COOLING RJ. Management of subluxated crystalline lens. Ophthalmology 99:542-545, 1992.

19 - DIETLEIN TS; JACOBI PC; KONEN W \& KRIEGLSTEIN GK. Complications of endocapsular tensin ring implantation in a child with Marfan's syndrome. J Cataract Refract Surg 26:937-940, 2000

20 - SYRDALEN P. Pars plana technique for removal of subluxated lenses in young patients. Acta Ophthalmol 65:585-588, 1987.

21 - GIRARD LJ; CANIZALES R; ESNAOLA N \& RAND WJ. Subluxated (ectopic) lenses in adults. Ophthalmology 97:462-465, 1990

22 - YU YS; KANG YH \& LIM KH. Improvements in visual acuity following limbal lensectomy for subluxated lens. Ophthalmic Surg Lasers 28:1006-1010, 1997.

23 - TSAI YY \& TSENG SH. Transscleral fixation of foldable intraocular lens after pars plana lensectomy for subluxated lens. J Cataract Refract Surg 25:722-724, 1999.

24 - ZETTERSTROM C; LUNDVALL A; WEBBER H JR \& JEEVES M. Sulcus fixation without capsular support in children. J Cataract Refract Surg 25:776-781, 1999.

25 - KAZEMI S; WIROSTKO WJ; SINHA S; MIELER WF; KOENIG SB \& SHETH BP. Combined pars plana lensectomy-vitrectomy with open loop flexible anterior chamber intraocular lens (AC IOL) implantatiom for subluxated lenses. Trans Am Ophthalmol Soc 98:247-251, 2000.

26 - LAM DSC; YOUNG AL; LEUNG ATS; RAO SK; FAN D \& NG JSK. Scleral fixation of a capsular tension ring for severe ectopia lentis. J Catarac Refract Surg 26:609-612, 2000.

Recebido para publicação em 17/09/2001

Aprovado para publicação em 07/03/2002 